\title{
Extract of Alpinia officinarum Suppresses Enteropathogenic Escherichia coli (EPEC) Lipopolysaccharide (LPS) Induced Inflammation in J774 A.1 Macrophages
}

\author{
Krishnan Subramanian, ${ }^{a, \#}$ Chinnasamy Selvakkumar, ${ }^{a, \#}$ Sankaranarayanan Meenakshisundaram, ${ }^{a}$ \\ Arun Balakrishnan, ${ }^{b}$ and Baddireddi Subhadra Lakshmi*, $a$ \\ ${ }^{a}$ Centre for Biotechnology, Anna University, Chennai-600025, Tamil Nadu, India and ${ }^{b}$ Nicholas Piramal Research Centre, 1, Nirlon \\ complex, Off Western Express Highway, Goregaon East, Mumbai-400063, India
}

(Received August 21, 2007; Accepted September 7, 2007)

Molecules with bi-functional activities are preferred targets in drug discovery research today. In this study, a possible dual role (anti-inflammatory and anti-bacterial activity) of a plant extract has been discussed. As an initiative, the extract is found to suppress inflammation caused by endotoxin release from an enteric pathogen. Enteropathogenic Escherichia coli $(E$. coli) (EPEC O127:H6) causes persistent diarrhea and continues to be a major health problem among infants in developing countries. Host inflammatory response during EPEC infection is weak when compared to infections caused by other enteric pathogens. This is due to the suppression of intestinal inflammatory response by type III secreted proteins of EPEC during infection process. The weak inflammatory response is thus possibly due to disruption of tight junctions, which in turn facilitate recruitment of macrophages from the underlying lamina propria or shedding of bacterial products like lipopolysaccharide (LPS) during antibiotic mediated bacterial lysis. Since intestinal cells are non-responsive to LPS, the inflammatory response would be elicited by the intestinal macrophages present at the site of infection. Apart from commercial LPS from E. coli O111: B4, LPS was isolated from EPEC and used for inducing inflammatory markers like TNF $\alpha, \mathrm{IL}-1 \beta$, IL-8 and TLR4 in J774 A.1 murine macrophage like cells to make this study clinically relevant. Alpinia officinarum is a traditional, perennial herb used widely in China and India for treating arthritis and gastrointestinal disorders. The ethyl acetate extract of Alpinia officinarum effectively suppressed EPEC LPS

\footnotetext{
*To whom correspondence should be addressed: Center for Biotechnology, Anna University, Chennai-600025, Tamil Nadu, India. Tel.: +91-44-22350772; Fax: +91-44-22350299; E-mail: lakshmibs@annauniv.edu

\#These authors contributed equally to this work.
}

induced inflammatory response thereby exhibiting potential in a clinical scenario, wherein diarrhea induced by EPEC or products of its lysis is either a consequence of macrophage induced inflammation or antibiotic treatment or due to a potential, yet to be analyzed, anti-bacterial activity of Alpinia officinarum extract against EPEC.

Key words__ Enteropathogenic Escherichia coli, inflammation, Alpinia officinarum, immunomodulator

\section{INTRODUCTION}

Enteropathogenic Escherichia coli (E. coli) (EPEC) is one of the leading causes of diarrhea in developing countries. ${ }^{1)}$ EPEC induces inflammation in host cells by activation of various inflammatory markers like NF- $\kappa \mathrm{B},{ }^{2)}$ ERK1/2, ${ }^{3)}$ PKC zeta ${ }^{4)}$ and transmigration of neutrophils. ${ }^{5)}$ Secretion of IL- 8 by host cells in response to EPEC infection helps in the recruitment of polymorphonuclear leucocytes. ${ }^{6}$ ) But there has been a contradicting report claiming that EPEC suppresses host immune responses ${ }^{7)}$ and it states that recruitment of polymorphonuclear leucocytes takes place at a later stage of EPEC infection, i.e., after tight junction disruption. However, the same study states that flagellin can cause basolateral stimulation of IL-8 in the intestine. It has also been reported that EPEC inhibits subsequent phagocytosis by recruited macrophages. ${ }^{8)}$ All the aforementioned studies pertain to the effect of whole bacteria on intestinal cells. But on the other hand, EPEC products like flagellin ${ }^{9)}$ and outer membrane preparations ${ }^{10}$ ) have been implicated as causes of inflammation in intestinal cells. Hence, there is a subtle difference in host responses to whole bacte- 
ria or its products wherein the host responds more quickly to bacterial products than the whole bacteria as such. Intestinal cells are unresponsive to either bacteria-associated or free LPS to prevent any non-specific immune response to LPS of normal bacterial flora in the intestine. But commercial preparations of LPS have been shown to induce inflammation in non-intestinal cell types like RAW 264.7 macrophages. ${ }^{11)}$ Commercial LPS preparations have been shown to contain traces of lipidassociated proteins, which is the possible reason for up-regulation of TLR2. When the protein contaminant is removed, the re-extracted LPS specifically induce TLR4 alone and not TLR2. ${ }^{12)}$ However, proteinase $\mathrm{K}$ digested bacterial whole cell lysates have proven to eliminate possible protein traces to a great extent in LPS preparations. ${ }^{13)}$ A possible way to test the efficacy of this method would be to check TLR4 up-regulation by both commercial LPS and LPS extracted using proteinase K digestion. Medicinal plants have been used from time immemorial for the treatment of various inflammatory disorders. Alpinia officinarum is a traditional, perennial herb of the Zingiberaceae family that is used widely in China and India for treating arthritis and gastrointestinal disorders. ${ }^{14)}$ In this study, we have examined the possible role of an ethyl acetate extract of Alpinia officinarum in modulating inflammation caused by LPS of EPEC on J774 A.1 cells.

\section{MATERIALS AND METHODS}

Chemical and Reagents — Cell culture medium (Rosewell Park Memorial Institute-1640 or RPMI$1640)$ supplemented with glutamine $(2 \mathrm{mM})$, and $10 \%$ heat-inactivated fetal calf serum was purchased from GIBCO BRL (Carlsbad, CA, U.S.A.). $\left[{ }^{3} \mathrm{H}\right]$ Thymidine was obtained from Amersham Pharmacia Biotech (Buckinghamshire, U.K.). Trizol reagent for RNA extraction was purchased from Sigma (Andover, U.K.). Moloney Murine Leukemia Virus (MMLV) reverse transcriptase and deoxyribonucleotide triphosphate (dNTP) and Thermus aquaticus (Taq) DNA polymerases for PCR were obtained from GIBCO BRL (Carlsbad, CA, U.S.A.), and New England Biolabs (Herts, U.K.), respectively. Phytohaemagglutinin (PHA) and LPS from E. coli serotype 0111:B4 were purchased from Sigma (Andover, U.K.). 3-(4,5-Dimethylthiazol-2yl)-2,5-diphenyltetrazolium bromide (MTT) assay kit was purchased from Promega (Madison, WI,
U.S.A.).

Collection of Plants and Preparation of Extracts - Alpinia officinarum rhizome was collected from Tamil Nadu Medicinal Corporation Limited (TAMCOL) medicinal garden at Kolli Hills, South India, India. The freshly collected rhizome was chopped, shadow dried and coarsely powdered by using a mixer grinder for 5-10 min. One hundred grams of coarse rhizome powder was extracted using organic solvents in increasing order of polarity (hexane, dichloromethane, ethyl acetate, methanol and water). The extracts were dried under reduced pressure using a rotary flash evaporator. The final yield was $2-4 \mathrm{~g}$ for each extract. The dried crude extracts were used for in vitro assays, dissolved in dimethyl sulfoxide (DMSO).

Cell Culture — The murine macrophage- like cell line, J774 A.1 (American Type Culture Collection, Manassas, VA, U.S.A.) was cultured in Dulbecco's Modified Eagle's Medium (DMEM) supplemented with $2 \mathrm{mM}$ glutamine, antibiotics (penicillin $120 \mathrm{U} / \mathrm{ml}$, streptomycin $75 \mu \mathrm{g} / \mathrm{ml}$, gentamycin $160 \mu \mathrm{g} / \mathrm{ml}$, and amphotericin B $(3 \mu \mathrm{g} / \mathrm{ml})$ and $10 \%$ heat-inactivated fetal calf serum (FCS). All cell cultures were maintained at $37^{\circ} \mathrm{C}$ in a humidified incubator with $5 \% \mathrm{CO}_{2}$.

Peripheral Blood Mononuclear Cell (PBMC) Isolation — PBMC were isolated as described. ${ }^{11)}$ Briefly, heparinized venous blood from healthy donors was obtained with their informed consent. Cells were obtained by density gradient centrifugation using ficoll histopaque. The buffy layer containing PBMC was carefully collected, washed twice with RPMI-1640 medium, and then the cells were counted. The cells were then seeded onto 96well $\mathrm{U}$ bottom plates at a density of $0.2 \times 10^{6}$ cells per well.

Mitogen-induced Lymphocyte Proliferation Assay — For the cell proliferation study, the peripheral blood mononuclear cells were separated as described. Triplicate cultures in $200 \mu \mathrm{l}$ of PBMC suspension were cultured in 96-well U bottom micro titer plates at $0.2 \times 10^{6}$ cells/well in RPMI medium containing $10 \%$ FCS and $1 \mu \mathrm{g} / \mathrm{ml}$ of PHA. The cells were treated with different concentrations of crude extracts (hexane, dichloromethane, ethyl acetate, methanol and water) and incubated for 3 days. After $72 \mathrm{hr}$, the cultures were pulsed with $\left[{ }^{3} \mathrm{H}\right]$ thymidine $(0.5 \mu \mathrm{Ci} /$ well $)$ during the last $16 \mathrm{hr}$ of incubation. The cells were then harvested and the radioactivity measured using a scintillation counter (Packard, Ramsey, MN, U.S.A.). The results are expressed as 
A

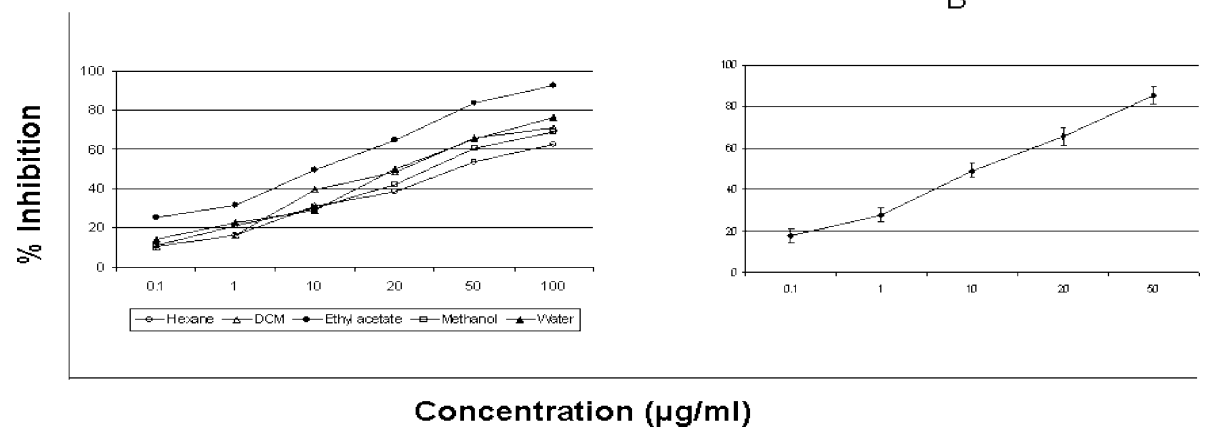

Fig. 1. A: Analysis of lymphocyte proliferation assay for Alpinia officinarum crude extract at $72 \mathrm{hr}$ on human PBMC. B: Doseresponse analysis of ethyl acetate crude extract in mitogen-stimulated PBMC. Dose-response study was performed for Alpinia officinarum crude ethyl acetate extract on mitogen-induced PBMC with different concentrations $(0.1 \mu \mathrm{g} / \mathrm{ml}, 1 \mu \mathrm{g} / \mathrm{ml}, 10 \mu \mathrm{g} / \mathrm{ml}$, $20 \mu \mathrm{g} / \mathrm{ml}$, and $50 \mu \mathrm{g} / \mathrm{ml}$ ). The optimum dose was found to be $20 \mu \mathrm{g} / \mathrm{ml}$. The results are expressed in terms of percentage inhibition with respect to stimulated control.

the mean \pm SEM.

\section{Lactate Dehydrogenase (LDH) Release As-} say — To confirm that the suppressive effect on lymphocyte proliferation was not due to cytotoxicity, the supernatants were also assayed for the enzyme LDH. This stable cytosolic enzyme, which is released upon cell lysis, was measured using the Cyto Tox $96^{\mathrm{TM}}$ assay.

Extraction of EPEC LPS — LPS was extracted according to the method of Hitchcock and Brown, ${ }^{13)}$ with slight modifications. Briefly, overnight cultures of EPEC grown on Luria-Bertani Agar were suspended in ice cold PBS ( $\mathrm{pH} 7$ ) and centrifuged at $10000 \mathrm{rpm}$ for $10 \mathrm{~min}$. The pellet was solubilized in $200 \mu \mathrm{l}$ of lysis buffer ( $20 \%$ glycerol, 5\% 2-Mercaptoethanol, 4.6\% sodium dodecyl sulfate (SDS) and $0.125 \mathrm{M}$ Tris- $\mathrm{HCl} \mathrm{pH} 6.8$ ) and heated at $100^{\circ} \mathrm{C}$ for $10 \mathrm{~min}$. The lysate was cooled to room temperature and $40 \mu \mathrm{l}$ of lysis buffer containing $2.5 \mathrm{mg} / \mathrm{ml}$ of proteinase $\mathrm{K}$ was added and the lysate was heated at $60^{\circ} \mathrm{C}$ for $1 \mathrm{hr}$. After boiling for 5 min, the sample was separated on $10 \%$ SDS-poly acrylamide gel electrophoresis (PAGE) and visualized by silver staining.

One $\mu \mathrm{g} / \mathrm{ml}$ of both commercial O111:B4 LPS and EPEC LPS were used for stimulating J774 A.1 cells $\left(1 \times 10^{6}\right.$ cells/well $)$ for a time period of $12 \mathrm{hr}$. To check the activity of the ethyl acetate extract, $20 \mu \mathrm{g}$ of extract was added to the cells $30 \mathrm{~min}$ after stimulation with EPEC LPS in a separate well. After $12 \mathrm{hr}$, the cells were harvested for RNA isolation.

Isolation of Total RNA and Semiquantitative Reverse Transcription-polymerase Chain Reaction (RT-PCR) — After the aforementioned time point of incubation, macrophages were immediately

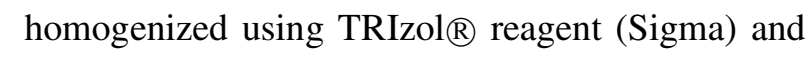
RNA was isolated by phenol-chloroform extraction. The aqueous phase containing RNA was then precipitated by adding an equal volume of isopropyl alcohol. The RNA obtained was then converted to cDNA by reverse transcription using MMLV reverse transcriptase enzyme and subjected to PCR with specific primers. PCR products were run on $1.2 \%$ agarose gels, stained with ethidium bromide and photographed. PCR products were consistent with the predicted sizes. PCR products were analyzed on ethidium bromide-stained agarose gels.

Statistical Analysis — All data are expressed as the mean \pm SEM. The statistical significance was evaluated by one-way analysis of variance (ANOVA) using Statistical Package for the Social Sciences (SPSS) version 10.0 (SPSS, Cary, NC, U.S.A.). When there was a significant difference, Tukey's multiple comparisons were performed by fixing the significance level at $p<0.05$.

\section{RESULTS}

\section{Ethyl Acetate Extract of Alpinia officinarum Shows Maximum Inhibition on Mitogen- Induced PBMCs}

Using thymidine incorporation assay, the ethyl acetate extract of Alpinia officinarum was found to show maximum inhibition of lymphocyte proliferation. The ethyl acetate extract showed maximum inhibition when compared to the other extracts (Fig. 1A). Significant inhibition of $65 \%$ of crude ethyl acetate extract was found at $20 \mu \mathrm{g} / \mathrm{ml}$ concentrations (Fig. 1B). The $20 \mu \mathrm{g} / \mathrm{ml}$ concentration of ethyl acetate was assessed for cytotoxicity 
by MTT assay and was found to be non-cytotoxic at the same time point of $72 \mathrm{hr}$ (data not shown).

\section{Ethyl Acetate Extract of Alpinia officinarum Suppressed EPEC LPS Induced Inflammatory Markers TNF $\alpha$, IL-1 $\beta$, IL-8 and TLR4}

EPEC LPS-induced expression of TNF $\alpha$, IL$1 \beta$, IL- 8 and TLR4 was suppressed by the extract. Expression of all the pro-inflammatory markers was assessed under conditions optimized with Glyceraldehyde-3-phosphate dehydrogenase (GAPDH). TLR4 expression was interestingly more up-regulated in EPEC LPS treated sample

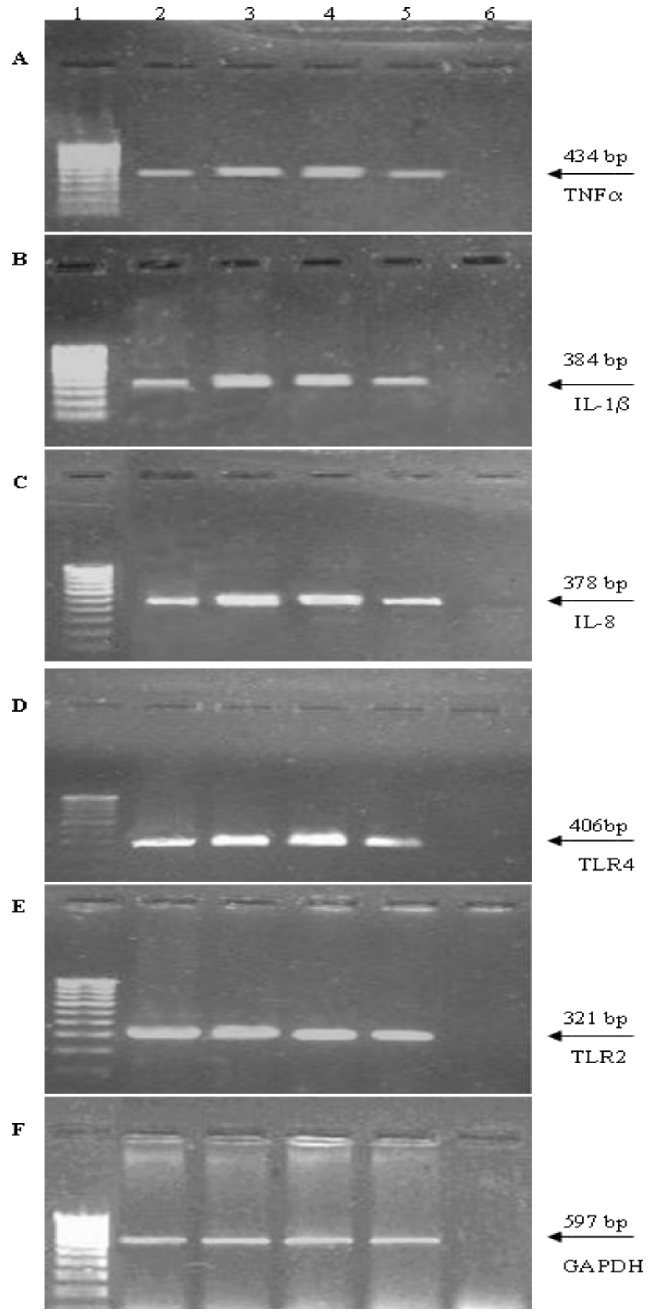

Fig. 2. Analysis of Alpinia officinarum Crude Extract on O111:B4 LPS and O127:H6 EPEC LPS-induced Pro-inflammatory Cytokine Expression in J774 A.1 Macrophages

A: TNF $\alpha$, B: IL-1 $\beta$, C: IL-8, D: TLR4, E: TLR2, F: GAPDH. J774 A. 1 cells were induced with LPS $(1 \mu \mathrm{g} / \mathrm{ml})$ at $12 \mathrm{hr}$ and the inhibitory effect of crude ethyl acetate extract on cytokine expression was studied by RT-PCR analysis. Lane 1: 100 bp Marker, Lane 2: Cell control, Lane 3: Cells + O111:B4 LPS, Lane 4: Cells+ O127:H6 EPEC LPS, Lane 5: Cells + O127:H6 EPEC LPS + Crude ethyl acetate extract $(20 \mu \mathrm{g} / \mathrm{ml})$, Lane 6: PCR negative control. when compared to commercial LPS-treated sample (Fig. 2).

\section{DISCUSSION}

Enteric pathogens employ various strategies to evade the immune system and persist in the intestine. The predominant ways are alterations in the structure and function of the tight junction barrier, induction of fluid and electrolyte secretion, and activation of the inflammatory cascade. ${ }^{15)}$ EPEC being a non-invasive pathogen has to persist in the intestinal surface and resist a variety of inflammatory responses. In this study, we observed that EPEC LPS stimulated a series of pro-inflammatory markers like TNF $\alpha$, IL- $1 \beta$, IL- 8 and TLR4. Similar observations have been reported earlier, wherein, spheroplasts and filaments of meropenem-treated $E$. coli produced more systemic and circulating endotoxins that untreated bacteria in experimental mice. Furthermore, the spheroplasts regained rod shape when the antibiotic was withdrawn. ${ }^{16)}$ This supports our hypothesis that endotoxins or LPS as such can induce high levels of $\mathrm{TNF} \alpha$ and other proinflammatory cytokines. This could also possibly explain the reason for persistent diarrhea that is typical of an EPEC infection. The initial stages of diarrhea might be due to electrolyte secretion as mentioned earlier and the later stages of the same might be due to endotoxin release and stimulation of pro-inflammatory cytokines in macrophages. Tolllike receptors play a major role in defense against invading pathogens. They are also important in the innate and adaptive immune responses of mammalian systems. ${ }^{17)}$ An interesting observation was that EPEC LPS prepared by proteinase K digestion induced higher TLR4 levels than commercial LPS. To support this observation, expression of TLR2 was analyzed. We found that TLR2 expression was at the basal level in both the control cells as well as EPEC LPS-treated cells. This indicates that EPEC LPS preparation had bare minimal protein contamination, if any. Commercial LPS from $E$. coli O111:B4 showed visibly significant mild upregulation of TLR2, which possibly might be due to trace protein contamination, although more input is required to quantitate its biological significance in EPEC pathogenesis. EPEC LPS preparation using proteinase $\mathrm{K}$ mediated cell lysis showed commendable specificity and could be used as an alternative for phenol-water extraction procedure. 
$\mathrm{TNF} \alpha$ and IL- $1 \beta$ play a major role in activating other cellular inflammatory markers and are also capable of self-stimulation. ${ }^{18)} \mathrm{TNF} \alpha$ also plays a major role in IL-8 stimulation in response to bacterial infection. Many medicinally important plants have shown immunomodulatory properties by suppressing pro-inflammatory cytokines. Recently, we reported that a methanolic extract of Ocimum basilicum suppressed pro-inflammatory cytokines in PBMC and RAW 264.7 macrophages. ${ }^{19)}$ Diarylheptanoids of Alpinia officinarum have been shown to suppress inflammatory cytokines in PBMCs and RAW 264.7 macrophages. ${ }^{20)}$ Also, ether and ethyl acetate extracts of Alpinia galanga of the Zingiberaceae family have been shown to possess antibacterial activity. ${ }^{21)}$ Alpinia species have also been shown to be safe as neutraceuticals in young children for the treatment of osteoarthritis. ${ }^{22)}$ These studies support the theory that extracts of Alpinia officinarum might possess dual anti-bacterial and antiinflammatory activities. Synergy experiments with the anti- malarial plant drug Artemisinin and antibiotics have been shown to reduce inflammation. ${ }^{23)}$ Identification of lead molecules that might show these dual properties is currently being assessed in our lab. These lead molecules could also be tested in conjunction with antibiotics to assess any probable synergistic activity in killing the bacteria or in suppression of inflammation. The pathogenic strategies of EPEC are such that even antibiotic treatment would still release its endotoxins and induce inflammation-induced diarrhea. Thus, novel molecules from a neutraceutically evaluated genus like Alpinia might not only be potentially active in their bactericidal activities against EPEC but also suppress the subsequent endotoxin mediated inflammation after bacterial lysis.

Acknowledgements We sincerely thank Lakshmi Janakarajan and Jothi Krithika for their critical suggestions.

\section{REFERENCES}

1) Clarke, S. C., Haigh, R. D., Freestone, P. P. E. and Williams, P. H. (2003) Virulence of Enteropathogenic E. coli, a global pathogen. Clin. Microbiol. Rev., 16, 365-378.

2) Savkovic, S. D., Koutsouris, A. and Hecht, G. (1997) Activation of NF-kappaB in intestinal epithelial cells by enteropathogenic Escherichia coli. Am.
J. Physiol., 273, C1160-C1167.

3) Savkovic, S. D., Ramaswamy, A., Koutsouris, A. and Hecht, G. (2001) EPEC-activated ERK1/2 participate in inflammatory response but not tight junction barrier disruption. Am. J. Physiol. Gastrointest. Liver Physiol., 281, G890-G898.

4) Savkovic, S. D., Koutsouris, A. and Hecht, G. (2003) PKCzeta participates in activation of inflammatory response induced by enteropathogenic E.coli. Am. J. Physiol. Cell Physiol., 285, C512C521.

5) Savkovic, S. D., Koutsouris, A. and Hecht, G. (1996) Attachment of a noninvasive enteric pathogen, enteropathogenic Escherichia coli, to cultured human intestinal epithelial monolayers induces transmigration of neutrophils. Infect. Immun., 64, 4480-4487.

6) Creasey, E. A., Delahay, R. M., Bishop, A. A., Shaw, R. K., Kenny, B., Knutton, S. and Frankel, G. (2003) CesT is a bivalent enteropathogenic Escherichia coli chaperone required for translocation of both Tir and Map. Mol. Microbiol., 47, 209-221.

7) Ruchaud-Sparagano, M.-H., Maresca, M. and Kenny, B. (2007) Enteropathogenic Escherichia coli (EPEC) inactivate innate immune responses prior to compromising epithelial barrier function. Cell. $\mathrm{Mi}$ crobiol., 9(8), 1909-21.

8) Goosney, D. L., Celli, J., Kenny, B. and Finlay, B. B. (1999) Enteropathogenic Escherichia coli Inhibits Phagocytosis. Infect. Immun., 67, 490-495.

9) Zhou, X., Giron, J. A., Torres, A. G., Crawford, J. A., Negrete, E., Vogel, S. N. and Kaper, J. B. (2003) Flagellin of enteropathogenic Escherichia coli stimulates interleukin- 8 production in T84 cells. Infect. Immun., 71, 2120-2129.

10) Malladi, V., Puthenedam, M., Williams, P. H. and Balakrishnan A. (2004) Enteropathogenic Escherichia coli outer membrane preparations induce iNOS by activation of NfkappaB and MAP kinases. Inflammation, 28, 345-353.

11) Gayathri, B., Manjula, N., Vinaykumar, K. S., Lakshmi, B. S. and Balakrishnan, A. (2007) Pure compound from Boswellia serrata extract exhibits anti-inflammatory property in human PBMCs and mouse macrophages through inhibition of TNF $\alpha$, IL- $1 \beta$, NO and MAP kinases. Int. Immunopharmacol., 7, 473-482.

12) Hirschfeld, M., Ma, Y., Weis, J. H., Vogel, S. N. and Weis, J. J. (2000) Repurification of Lipopolysaccharide Eliminates Signaling Through Both Human and Murine Toll-Like Receptor-2. J. Immunol., 165, 618-622.

13) Hitchcock, P. J. and Brown, T. M. (1983) 
Morphological heterogeneity among Salmonella lipopolysaccharide chemotypes in silver-stained polyacrylamide gels. J. Bacteriol., 154, 269-277.

14) Wu, T. L. and Larsen, K. (2000) Family Zingiberaceae. In Flora of China, (Wu, Z. G. and Raven, P. H. Eds.), Science Press, Beijing, China, and Missouri Botanical Garden Press, St. Louis, Missouri, U.S.A., vol.24, pp.322-377.

15) Berkes, J., Viswanathan, V. K., Savkovic, S. D. and Hecht, G. (2003) Intestinal epithelial responses to enteric pathogens: effects on the tight junction barrier, ion transport, and inflammation. Gut, 52, 439451.

16) Buijs, J., Dofferhoff, A. S. M., Mouton, J. W. and van der Meer, J. W. M. (2006) Pathophysiology of in-vitro induced filaments, spheroplasts and rodshaped bacteria in neutropenic mice. Clin. Microbiol. Infect., 12(11), 1105-1111.

17) Heine, H. and Lien, H. (2003) Toll-like receptors and their function in innate and adaptive immunity. Arch. Allergy Immunol., 130, 180-192.

18) Dinarello, C. A. (2001) Proinflammatory cytokines, Chest, 118, 503-508.

19) Selvakkumar, C., Gayathri, B., Vinay Kumar, K. S., Lakshmi, B. S. and Balakrishnan, A. (2007) Po- tential Anti-inflammatory Properties of Crude Alcoholic Extract of Ocimum Basilicum L. in Human Peripheral Blood Mononuclear Cells. J. Health Sci., 53, 500-505.

20) Yadav, P. N., Liu, Z. and Rafi, M. M. (2003) A Diarylheptanoid from Lesser Galangal (Alpinia officinarum) Inhibits Proinflammatory Mediators via Inhibition of Mitogen-Activated Protein Kinase, p44/42, and Transcription Factor Nuclear Factor-KB J Pharmacol. Exp. Ther, 305(3), 925-31.

21) Thomas, E., Shanmugam, J. and Rafi, M. M. (1996) Antibacterial activity of plants belonging to Zingiberaceae family. Biomedicine, 16, 15-20.

22) Ameye, L. G. and Chee, W. S. S. (2006) Osteoarthritis and nutrition. From nutraceuticals to functional foods: a systematic review of the scientific evidence. Arthritis Research \& Therapy., 8:R127.

23) Wang, J., Zhou, H., Zheng, J., Cheng, J., Liu, W., Ding, G., Wang, L., Luo, P., Lu, Y., Cao, H., Yu, S., Li, B. and Zhang, L. (2006) The Antimalarial Artemisinin Synergizes with Antibiotics To Protect against Lethal Live Escherichia coli Challenge by Decreasing Proinflammatory Cytokine Release. Antimicrobial Agents Chemother, 50, 2420-2427. 